\title{
Good Laboratory Practice Type
}

National Cancer Institute

\section{Source}

National Cancer Institute. Good Laboratory Practice Type. NCI Thesaurus. Code C90389.

A classification of the good laboratory practices employed in a study. 